\title{
Postnatal Transplantation of Interneuronal Precursor Cells Decreases Anxiety-Like Behavior in Adult Mice
}

\author{
M. F. Valente, ${ }^{*}$ S. Romariz, ${ }^{*}$ M. E. Calcagnotto, $\dagger$ L. Ruiz, ${ }^{*}$ L. E. Mello, ${ }^{*}$ R. Frussa-Filho $\ddagger$ and B. M. Longo* \\ *Department of Physiology, Universidade Federal de São Paulo, São Paulo, Brazil \\ $\dagger$ Department of Biochemistry, Universidade Federal do Rio Grande do Sul, Porto Alegre, Brazil \\ †Department of Pharmacology, Universidade Federal de São Paulo, São Paulo, Brazil
}

\begin{abstract}
The GABAergic system is critically involved in the modulation of anxiety levels, and dysfunction of GABAergic neurotransmission appears to be involved in the development of generalized anxiety disorder. Precursor cells from the medial ganglionic eminence (MGE) have the ability to migrate and differentiate into inhibitory GABAergic interneurons after being transplanted into the mouse brain. Thus, transplantation of interneuronal precursor cells derived from the MGE into a postnatal brain could modify the neuronal circuitry, increasing GABAergic tone and decreasing anxiety-like behavior in animals. Our aim was to verify the in vivo effects of transplanted MGE cells by evaluating anxiety-like behavior in mice. MGE cells from 14-day green fluorescent protein (GFP) embryos were transplanted into newborn mice. At 15, 30, and 60 days posttransplant, the animals were tested for anxiety behavior with the elevated plus maze (EPM) test. Our results show that transplanted cells from MGE were able to migrate to different regions of the brain parenchyma and to differentiate into inhibitory interneurons. The neuronal precursor cell transplanted animals had decreased levels of anxiety, indicating a specific function of these cells in vivo. We suggested that transplantation of MGE-derived neuronal precursors into neonate brain could strengthen the inhibitory function of the GABAergic neuronal circuitry related to anxiety-like behavior in mice.
\end{abstract}

Key words: GABAergic neuron (GABA); Medial ganglionic eminence (MGE); Green fluorescent protein (GFP); Cell differentiation; Anxiolysis

\section{INTRODUCTION}

The medial and caudal ganglionic eminences (MGE, CGE) of the ventral telencephalon in the embryonic brain give rise to the majority of the cerebral cortex's inhibitory interneurons $(25,40-42)$. In the developing brain, these cells have the potential to migrate from these regions and integrate into the circuitry of the striatum, cortex, hippocampus, and olfactory bulb (40). When transplanted into a neonate brain, MGE-derived precursor cells preserve their ability to migrate and differentiate into $\gamma$-aminobutyric acidergic (GABAergic) interneurons, establishing functional inhibitory synapses with preexisting neurons and modifying the inhibitory tone in the host brain $(1,5,40)$.

It is widely believed that GABAergic circuits are critically involved in the modulation of anxiety levels (28). A reduction in GABAergic neurotransmission could contribute to the emergence of anxiety disorders (2). Moreover, the anxiolytic effect of drugs that act on the GABA receptors (32) or the engraftment of GABAergic cells into the amygdaloid nuclei (11) provides strong evidence that GABA dysfunction underlies anxiety states. Currently, investigations are being intensively conducted to better understand the GABAergic circuits that are involved in the regulation of anxiety levels and mood disorders. The amygdala has been considered to be directly involved in anxiety and the emotion of fear since the early studies of Kluver and Bucy (22 for review). However, investigations over the last two decades have produced advances toward determining the neuroanatomy of other circuits related to the behavior of fear and anxiety, which connect the amygdala with other regions of the brain $(33,36)$. Studies have shown that, in addition to the amygdala, the temporal and prefrontal cortices also play a critical role in the expression of anxiety and fear conditioning $(3,4,6,16,17,21,27)$.

The focus of this study was to investigate the effects of transplanted cells in vivo in three different phases of development. We hypothesized that interneuronal precursor cells transplanted into the immature brain could integrate

Received August 11, 2011; final acceptance June 22, 2012. Online prepub date: October 1, 2012.

Address correspondence to Beatriz Monteiro Longo, Neurofisiologia, Depto. Fisiologia - UNIFESP - SP, R. Botucatu, 8625 andar,

V. Clementino - São Paulo - SP, Brazil. Tel: (5511) 5579-2033 or 5576-4513; E-mail: beatriz.longo@ unifesp.br or beatrizlongo@gmail.com 
into the host neural circuitry and have inhibitory effects that would influence the overall level of anxiety in mice throughout the maturation of the central nervous system. Since its introduction by Handley and Mithani (18) and its experimental validation for rats (30) and mice (23), the elevated plus maze (EPM) has been most extensively used to assess the anxiolytic and anxiogenic effects of drugs and to measure levels of spontaneous anxiety-like behavior $(10,20,31)$. In the present study, we evaluated the EPM behavior of mice that were neonatally transplanted with MGE-derived cells. The test was performed at three different times for posttransplantations $(15,30$, and 60 days). GABAergic neurotransmission is also involved in memory (24) and motor function (19), and so the behavior of transplanted mice was also quantified in the passive avoidance memory task, and their general activity was quantified in the open-field test. Immunohistochemistry was also performed to confirm the ability of precursor cells from the MGE to migrate and differentiate into inhibitory GABAergic interneurons after being transplanted into the mouse brain.

\section{MATERIALS AND METHODS}

All of the animals were housed in a pathogen-free facility and were maintained in accordance with the Guide for the Care and Use of Laboratory Animals (National Research Council). Experimental protocols were approved by the Animal Care and Ethics Committee of UNIFESP/SP.

\section{MGE Extraction and Transplantation}

Transgenic mice expressing enhanced green fluorescent protein (GFP) cDNA under the control of a chicken $\beta$-actin promoter and cytomegalovirus enhancer, provided by CEDEME (Center for the Development of Animal Models in Biology and Medicine at Universidade Federal de São Paulo, São Paulo, Brazil), were used as MGE donors. For tissue extraction and cell dissociation, ventricular and subventricular layers of MGE of E13.5 GFP transgenic mouse embryos were dissected. The tissue was removed and mechanically dissociated by pipetting and centrifugation; the cells were washed with medium [Dulbecco's modified Eagle's medium (DMEM); Gibco, Grand Island, NY, USA] containing DNase I (10 mg/ml; Invitrogen, Carlsbad, CA, USA); they were then centrifuged and resuspended in 4-5 $\mu$ l of cold culture medium. We determined the cell number and viability by the trypan blue (Gibco) exclusion method. The cell suspension was placed in a Narishige microinjector (Narishige Scientific Intrument Laboratory, Tokyo, Japan) guided with a stereotaxic apparatus. Neonatal C57/B16 mice [P3; CEDEME (Centro de Desenvolvimento de Modelos Experimentais em Medicina e Biologia) UNIFESP, São Paulo - SP, Brazil] $(n=28)$ were anesthetized by hypothermia $\left(-4^{\circ} \mathrm{C}\right)$, and $5 \times 10^{4}$ to $1.5 \times 10^{5}$ of the cells in a $0.4-\mu l$ volume were bilaterally injected at a $45^{\circ}$ angle into the neocortex (layers 3-4) of each mouse. The cell infusion was made in the superficial layers of neocortex (up to fourth layer) and not directly into brain structure that are commonly believed to be associated with anxiety, such as amygdala, to avoid track lesion in profound structures and further brain damage. Within this context, the migratory potential of these cells and their effective dispersion throughout the neocortex have been demonstrated $(1,5,40,42)$. The age-matched control group was injected with medium $(n=26)$ or with a similar volume of dead cells (by repeated frost and thaw cycles) as a control for the inflammatory response or GFP toxicity $(n=24)$.

\section{Behavioral Tests}

The behavioral tests were performed at 15, 30, and 60 days after transplantation with MGE cells (respectively, $n=9, n=6$, and $n=13)$, medium $(n=9, n=6$, and $n=11)$, or dead cells $(n=6, n=9$, and $n=9)$. The animals were evaluated using EPM tests and open-field (OF) tests at each time point, whereas the passive avoidance (PA) test was performed only at 60 days. The tests were performed during the light period (14:00-17:00 h). The behavior of each animal was videotaped by a video camera located $150 \mathrm{~cm}$ above the apparatus. Videotapes were later watched and analyzed by a trained observer. All of the observations were conducted blindly.

\section{Elevated Plus Maze (EPM) Test}

The Elevated Plus Maze (EPM; AVS, Solução Integrada Comercial, São Carlos-SP, Brazil) apparatus consisted of two open $(28.5 \times 7 \mathrm{~cm})$ and two enclosed $(28.5 \times$ $7 \times 14 \mathrm{~cm}$ ) arms made of wood, which were arranged in such a way that the two pairs of identical arms were opposite to each other. The open arms were bounded by 1-cm-high ledges on the border of the arms. The maze was raised to a height of $50 \mathrm{~cm}$ above the floor. Each mouse was placed in the center of the apparatus, and we recorded the number of entries into the open arms and the number of entries into the closed arms. In addition, we recorded the time spent in the open arms and the time spent in the closed arms. The percentage of time spent in the open arms and the entries into the open arms [i.e., (the time in or entries into the open arms)/(the time in or entries into the open arms + the time in or entries into the closed arms)] were recorded as anxiety-like behavior indexes, and the number of entries into either arm of the apparatus was used as an index of general activity.

\section{Open-Field (OF) Test}

The open-field (OF; AVS) apparatus consisted of a circular arena $85 \mathrm{~cm}$ in diameter, divided into 25 segments of approximately equal area by black painted lines on the 
white floor. The arena was bound by a wall $50 \mathrm{~cm}$ high. The central zone was defined as the central compartment of the floor, which was centrally located $6 \mathrm{~cm}$ from the perimeter of the chamber walls. The parameters assessed for the present studies were the frequency of entering the center and the time spent in the center and in the periphery. Each mouse was individually placed in the center of the OF apparatus, and the ambulation scores (the number of areas entered with all four paws) were tallied for $5 \mathrm{~min}$. Fecal pellets were removed, and the floor was wiped with clean damp tissues after every occupation. The motor activity was calculated using the distance moved in the OF. All of the testing was conducted under standard room lighting.

\section{Passive Avoidance (PA) Test}

The passive avoidance (PA; AVS) apparatus consisted of a dark compartment and an illuminated compartment (both measuring $10 \mathrm{~cm} \times 10 \mathrm{~cm}$ in an area with a wall height of $20 \mathrm{~cm}$ ) that were separated by a guillotine door. On the first day, the animal was placed in the illuminated compartment for an acclimatization period of $30 \mathrm{~s}$, and then the door was opened. As the mouse entered the dark compartment (four paws), the door was closed and a single low-intensity foot shock $(0.5 \mathrm{~mA} ; 5 \mathrm{~s})$ was delivered through the stainless steel rods. The mouse was then removed from the darkened chamber $10 \mathrm{~s}$ after the shock. The session was repeated at 90-min time intervals from the first training trial. Twenty-four hours later, the mouse was again placed in the illuminated compartment, and the latency to enter the dark chamber was measured, with a cutoff at $300 \mathrm{~s}$. Mice that did not enter into the dark compartment within $300 \mathrm{~s}$ were removed from the apparatus and assigned a latency score of $300 \mathrm{~s}$. A shock was not delivered in the retention trial. The time taken for a mouse to enter the dark compartment after the door opened was defined as the latency time for both the acquisition and the retention trials (37).

\section{Immunohistochemistry}

At the end of the behavioral tests, the animals were deeply anesthetized with $10 \%$ chloral hydrate $(400 \mathrm{mg}$ / kg, IP; Merck, Rio de Janeiro- RJ, Brazil) and perfused through the heart with $50 \mathrm{ml}$ of phosphate-buffered saline (PBS, Sigma-Aldrich, St. Louis, MO, USA) followed by $200 \mathrm{ml}$ of $4 \%$ paraformaldehyde (Synth, São Paulo - SP, Brazil) at $4^{\circ} \mathrm{C}$. Coronal brain cryostat sections $(35 \mu \mathrm{m}$ thick) were made between bregma 0.98 and bregma $-3.28 \mathrm{~mm}$ according to the stereotaxic coordinates of the mouse brain atlas (29). To visualize transplanted MGE cells, slices were processed for immunofluorescence for GFP and were double-labeled with interneuronal markers [neuropeptide Y (NPY), calretinin (CR), and parvalbumin (PV)]. The sections were incubated for
$2 \mathrm{~h}$ with an anti-GFP AlexaFluor 488-conjugated antibody (1:600; Molecular Probes/Invitrogen, Eugene, OR, USA), followed by an overnight incubation with rabbit antibodies against NPY (1:1,500, Molecular Probes), CR (1:2,000, Molecular Probes), and PV (1:2,000, Molecular Probes). The sections were then incubated with anti-rabbit IgG AlexaFluor 546-conjugated antibodies (1:600, Molecular Probes/Invitrogen) for $1 \mathrm{~h}$. All of the sections were mounted using a nuclear-counterstaining, fluorescencepreserving mounting medium containing DAPI (Vector Laboratories, Burlingame, CA, USA). The slides were examined using fluorescence (Nikon 80i; Nikon, Melville, NY, USA) and confocal (Olympus FluoView 1000; Olympus, Center Valley, PA, USA) microscopes. In each section, double-labeled cells were qualitatively evaluated for their localization in the brain areas of interest and for their morphology.

The estimation of GFP cells was sampled in five coronal sections for the neocortex, striatum, hippocampus, amygdala, thalamus, and corpus callosum. In counted slices, cells could be detected at $\sim 1 \mathrm{~mm}$ from the site of injection in adult animals (P30 and P60). Images of 10 random, nonoverlapping fields were captured and quantified for each region. The same counting protocol was used to estimate the percentage of double-stained $\mathrm{GFP}^{+} / \mathrm{NPY}^{+}$, $\mathrm{GFP}^{+} / \mathrm{CR}^{+}$, and $\mathrm{GFP}^{+} / \mathrm{PV}^{+}$cells. All of the images were captured and digitized using the Nikon ACT-1 v.2 system or the Olympus OLYMPUS FLUOVIEW Ver.2.0b Viewer (FV10-ASW) software.

\section{Statistical Analyses}

Statistical analysis of the behavior was performed using analysis of variance (ANOVA) followed by Tukey's multiple comparison post hoc test (GraphPad Prism v. 4.0, GraphPad Software, Inc., San Diego, CA, USA) to produce a comparison between MGE-transplanted, nontransplanted, and "dead cells" animals. A significance level of $5 \%$ was assumed.

\section{RESULTS}

\section{Anxiety-Like Behavior}

The anxiety-like behavior of the animals was evaluated by the EPM test at three different time points after transplantation (MGE15, MGE30, and MGE60) and compared to age-matched medium-injected (CTRL) and dead cell-injected (DC) animals (CTRL15, CTRL30, CTRL60 and DC15, DC30, DC60, respectively) (Fig. 1A, B). At 15 days posttransplantation, there were no significant differences in the percentage of entries and the time spent in the open arms when compared with the CTRL and DC groups), although there was a trend toward an increase in the percentage of time spent in the open arms at this age posttransplantation $(p=0.0893)$. Significant differences 
$\%$ open arms (s)
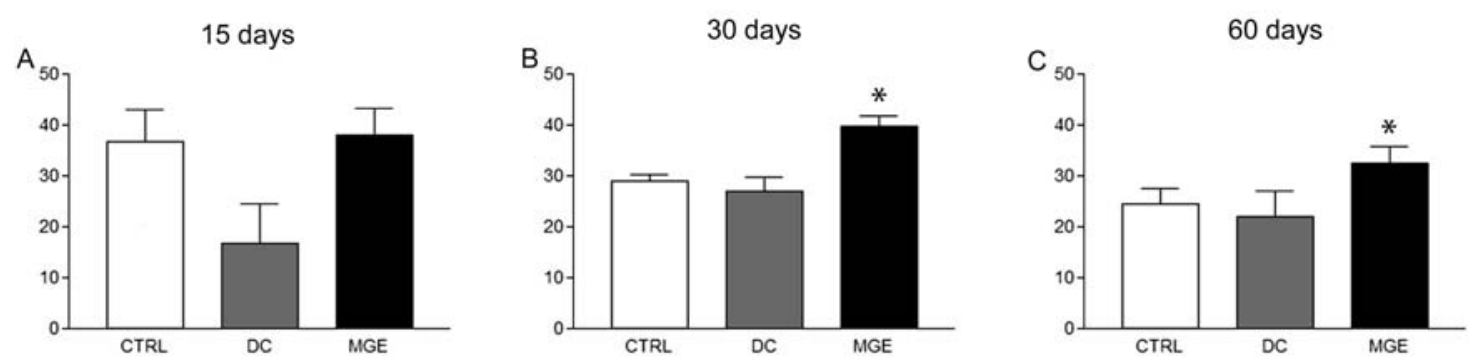

$\%$ entries in open arms
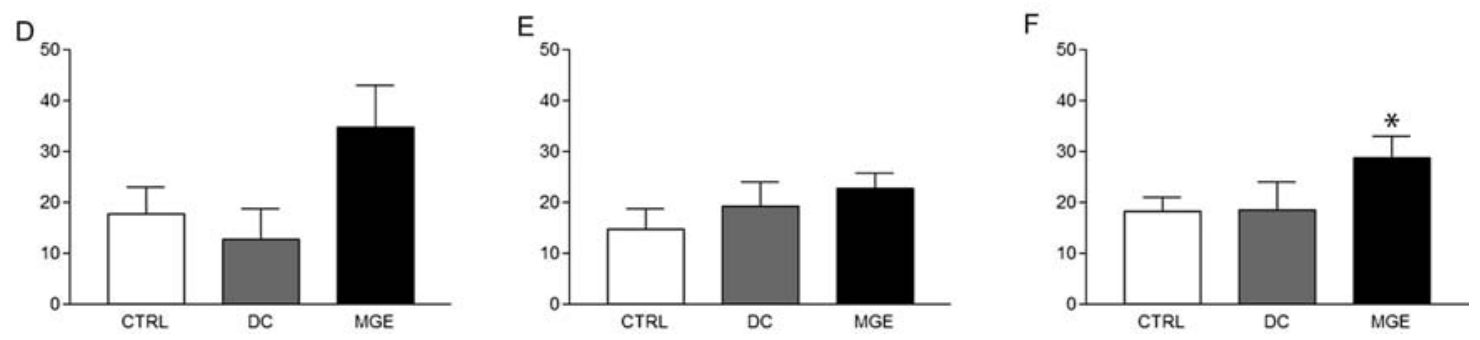

Figure 1. Elevated plus maze behavior of the control (CTRL), dead cell-transplanted (DC), and medial ganglionic eminence (MGE)transplanted (MGE) animals. The percentage of time spent in the open arms (A-C) and the percentage of entries in the open arms (D-F) at 15, 30, or 60 days after transplantation. MGE-transplanted mice entered the open arms more at 30 and 60 days after transplantation and spent significantly more time in the open arms at 60 days after transplantation. ${ }^{*} p<0.05$ compared with control and dead cell groups; one-way ANOVA, followed by post hoc Tukey’s multiple comparison test.

were detected at 30 and 60 days posttransplantation, indicating a reduction in anxiety levels in the MGEtransplanted animals when compared to the other groups. Thirty days after transplantation, there was a reducedanxiety behavioral pattern in the MGE30 group, which was indicated by a significant increase in the percentage of time spent in the open arms ( $p=0.0004)$ (Fig. 1B). At 60 days after transplantation (MGE60 group), there was a significant increase in the percentage of time spent the open arms $(p=0.0086)$ and entries in the open arms $(p=0456)$ (Fig. 1C, F). Concerning the total number of entries in both the open and closed arms, there were no significant differences at the three evaluated time points after transplantation. Closed arm entries has been proposed as an alternative index of general activity in the plus maze $(15,34)$, so this parameter was also quantified, but no significant differences were revealed at any of the time points (data not shown).

\section{Motor Activity and Memory Test}

The motor activity of the transplanted mice was assessed using the open-field test. There was no significant difference in the general activity of the MGE animals for any of the parameters that were examined. Indeed, the total distance traveled in $5 \mathrm{~min}$ was similar for the three groups, and in addition, there was no significant difference in the number of visited squares and the time spent in the center of the OF between the groups at the three different time points.

The PA test (a memory test) is based on the principle that performing certain behaviors leads to an unpleasant consequence; thus, the animal will avoid the repetition of that behavior, unless it has a memory deficit. There was no memory deficit measured by the acquisition test $(90 \mathrm{~min}$ after training) or the retention test ( $24 \mathrm{~h}$ after training) in the MGE-transplanted animals at 60 days posttransplantation when compared to CTRL animals. The percentage of animals that reached the cutoff score (300 s) at both $90 \mathrm{~min}$ and $24 \mathrm{~h}$ after the training was $75 \%$ and $42 \%$, respectively, in MGE60 animals and 50\% in the CTRL animals. We noted an increase, although it was not statistically significant, in the latency before entering the dark compartment for the MGE60 animals when they were tested 90 min after training $(p=0.074)$. We did not test animals at 15 and 30 days because of the aversive nature of the test.

The anxiolytic behavioral pattern of the MGEtransplanted animals was not likely the result of locomotor hyperactivity because the total number of entries and the open-field activity revealed no differences between the groups. The assessment of memory by the PA test indicated that there was no change in the short- or longterm memories between the groups. These findings favor 
the view that transplanted animals explored the maze with a normal pattern of motor activity, with no interference in memory processes.

\section{Inhibitory Neurons}

Qualitative evaluation of MGE-transplanted cells in the host brain was performed by immunohistochemistry to confirm the presence of $\mathrm{GFP}^{+}$transplanted cells in the brain parenchyma and to verify their inhibitory identity by colocalization with the interneuronal markers NPY, CR, and PV. The immunohistochemistry analysis indicated that cells that were transplanted into neonate animals had survived in the young (at 15 days) and adult hosts brains at 30 and 60 days $\left(\mathrm{GFP}^{+}\right.$cells with neuronal morphology) migrated throughout the parenchyma (found in different areas) and differentiated into inhibitory neurons (Figs. 2-4). GFP ${ }^{+}$cells found in the brain areas of MGE15 animals were poorly ramified and showed thin, short processes, whereas $\mathrm{GFP}^{+}$cells from the MGE30 (Fig. 3A-C) and MGE60 (Fig. 4A-C) animals displayed thicker and longer ramifications of cell processes compared to the MGE15 animals (Fig. 2A-C).

In the MGE15 groups, GFP cells were mainly found in the neocortex, striatum, hippocampus, and corpus callosum. The high number of $\mathrm{GFP}^{+}$cells in the corpus callosum suggests a preferential migratory path of these cells at this age, which is indicative of their undifferentiated stage. At this age, few $\mathrm{GFP}^{+}$cells colabeled for NPY, PV, and CR were found distributed in clusters (NPY and PV) (Fig. 2D-I) or scattered (CR) (Fig. 2J-L). At the evaluated time points 30 and 60 days after transplantation (MGE30 and MGE60 ), $\mathrm{GFP}^{+}$cells could be found mainly in the neocortex, striatum, septal area (medial septum), hippocampus (CA1 and dentate gyrus), corpus callosum, periventricular zone (ependymal area), thalamus, and amygdala. In MGE30 animals, GFP cells presented neuronal morphology, with long processes (Fig. 3A-C), and many of them expressed interneuronal markers for NPY, CR, and PV (Fig. 3F, I, L). In the MGE60 group, the GFP cells are clearly identified as mature inhibitory cells, and the majority were doublestained for NPY, CR, and PV (Fig. 4F, I, L).

An estimation of GFP cells in five main regions as well as double-staining for interneuronal markers was performed. From the total amount of GFP cells counted in the adult animals (P30 and P60), a high percentage of $\mathrm{GFP}^{+}$cells were found in the hippocampus, neocortex, and striatum $(38.7 \%, 35.4 \%$, and $17 \%$, respectively). Lower percentages of $\mathrm{GFP}^{+}$cells were found in the amygdala and thalamus (respectively, 6.7\% and 3\%) (Table 1). Although the site of injection was confirmed to be in the neocortex layer 3 or 4 , clusters of $\mathrm{GFP}^{+}$cells were found in the corpus callosum, corresponding to $24 \%$ of the total $\mathrm{GFP}^{+}$ cells counted. Substantial percentages of double labeling for GFP and inhibitory markers NPY, CR, and PV were observed in the hippocampus, neocortex, and striatum (respectively, 33.3\%, 30.8\%, and 28.3\%) of the adult animals. A smaller percentage of $\mathrm{GFP}^{+}$colabeled cells were found in the amygdala (6.3\%) and the thalamus (1.26\%). The total percentage of $\mathrm{GFP}^{+} / \mathrm{NPY}^{+}$was $51.6 \%$, the total percentage of $\mathrm{GFP}^{+} / \mathrm{CR}^{+}$was $28.3 \%$, and the total percentage of $\mathrm{GFP}^{+} / \mathrm{PV}^{+}$was $20.1 \%$. The percentage of $\mathrm{GFP}^{+} /$ $\mathrm{PV}^{+}$was $24.4 \%$ in the neocortex, $35.4 \%$ in the striatum, $28 \%$ in the hippocampus, $2.4 \%$ in the thalamus, and $9.7 \%$ in the amygdala. The percentage of $\mathrm{GFP}^{+} / \mathrm{CR}^{+}$was $40 \%$ in the neocortex, $17.8 \%$ in the striatum, $40 \%$ in the hippocampus, and $2.2 \%$ in the amygdala. Of the $\mathrm{GFP}^{+} /$ $\mathrm{NPY}^{+}$cells, $34.4 \%$ were in the neocortex, $25 \%$ in the striatum, $37.5 \%$ in the hippocampus, and $3.1 \%$ in the amygdala. $\mathrm{No} \mathrm{GFP}^{+} / \mathrm{CR}^{+}$or $\mathrm{GFP}^{+} / \mathrm{NPY}^{+}$cells were found in the thalamus (Table 1).

\section{DISCUSSION}

The main finding of this study is that transplantation of MGE interneuronal precursor cells into newborn mouse brains leads to anxiolytic effects in adulthood. Two months after the transplantation of MGE cells into postnatal animals, the transplanted cells survived, migrated throughout the host brain parenchyma and differentiated into inhibitory GABAergic interneurons. MGE-transplanted cells were detected in the hippocampus, neocortex, striatum, septal area, corpus callosum, periventricular zone (ependymal area), amygdala, and thalamus. Our results confirm the previous findings of migration and differentiation of MGE-transplanted cells into inhibitory interneurons in postnatal animals and add new data about its behavioral consequences in vivo.

We did not intend to use an anxiogenic model or perform a treatment to decrease anxiety levels by introducing MGE precursors in all of the regions involved in anxiety. Instead, we questioned whether cortical transplantation of MGE cells in newborn animals would affect anxietylike behavior and whether an age-dependent effect exists. The well-described $(1,5,42)$ migratory potential of these cells and their effective dispersion throughout the neocortex justified the experimental design that is delineated here. It would be important to know whether this type of graft procedure, which has been used by others in animal models of epilepsy $(5,9,13,39,43)$, Parkinson's disease (26), and stroke (12), could result in behavioral changes such as anxiolysis.

Studies using grafts of GABAergic precursors derived from embryonic telencephalic regions did not consider the excitatory role of GABA during development (7). Early in development, GABA acting via chloride-permeable $\mathrm{GABA}_{\mathrm{A}}$ channels has depolarizing and excitatory effects that result from elevated intracellular chloride concentrations in immature neurons (8). We did not observe any changes in the anxiety parameters of mice 15 days after 

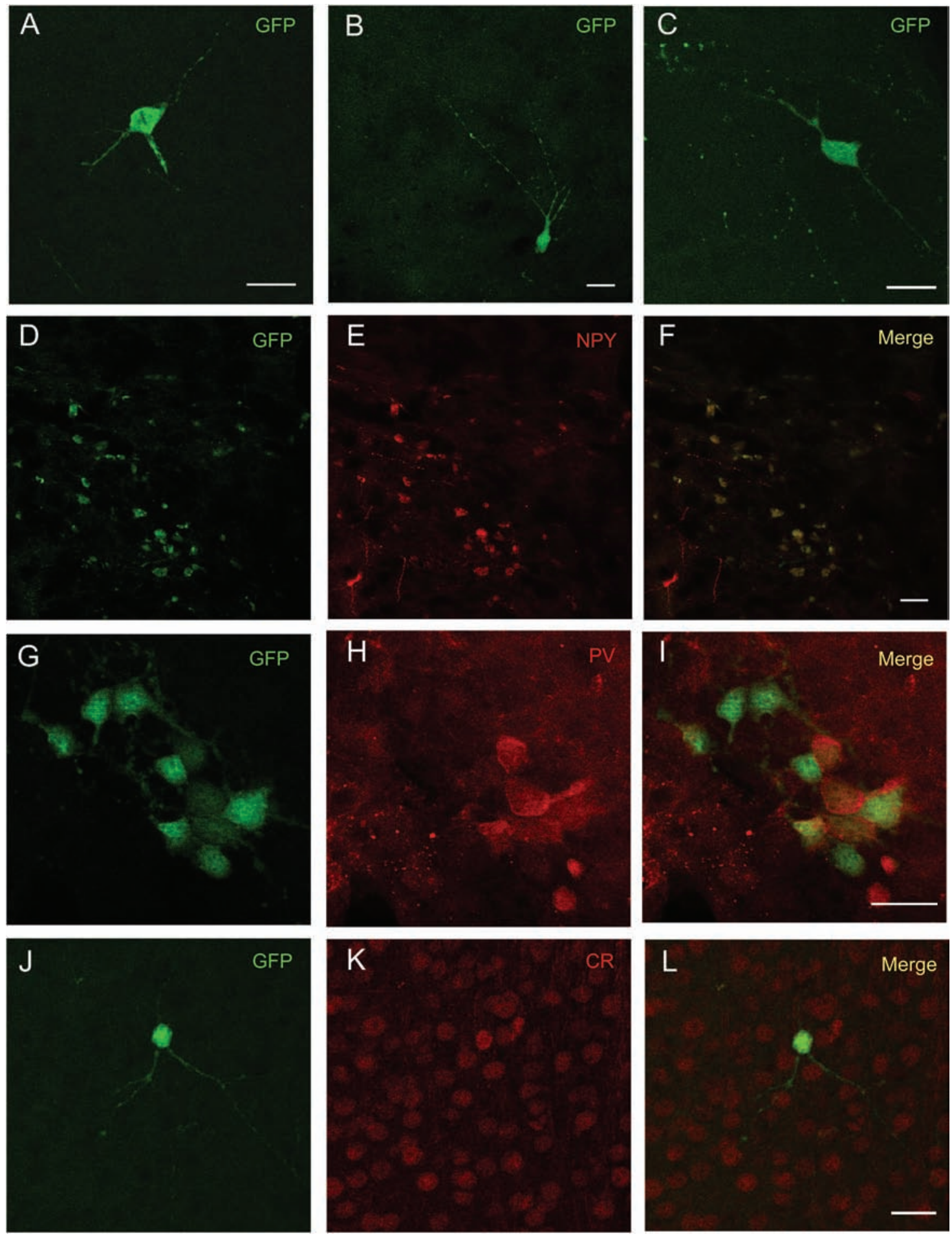

Figure 2. Morphology of MGE-derived $\mathrm{GFP}^{+}$cells 15 days after transplantation. MGE-derived GFP ${ }^{+}$cells 15 days after transplantation (A-C) in the hippocampus (A), striatum (B), and periventricular area (C). Double-stained immunohistochemical analysis of grafted $\mathrm{GFP}^{+}$cells (green) with interneuron-specific markers (red), colocalization of GFP ${ }^{+}$and $\mathrm{NPY}^{+}(\mathrm{D}-\mathrm{F}), \mathrm{GFP}^{+}$and $\mathrm{PV}^{+}(\mathrm{G}-\mathrm{I})$, and $\mathrm{GFP}^{+}$and $\mathrm{CR}^{+}(\mathrm{J}-\mathrm{L})$, respectively, in the neocortex $(\mathrm{D}-\mathrm{F}, \mathrm{J}-\mathrm{L})$ and periventricular area $(\mathrm{G}-\mathrm{I})$ is demonstrated in the merged images (F, I, L). Scale bars: $20 \mu \mathrm{m}$. GFP, green fluorescent protein; NPY, neuropeptide Y; PV, parvalbumin; CR, calretinin. 

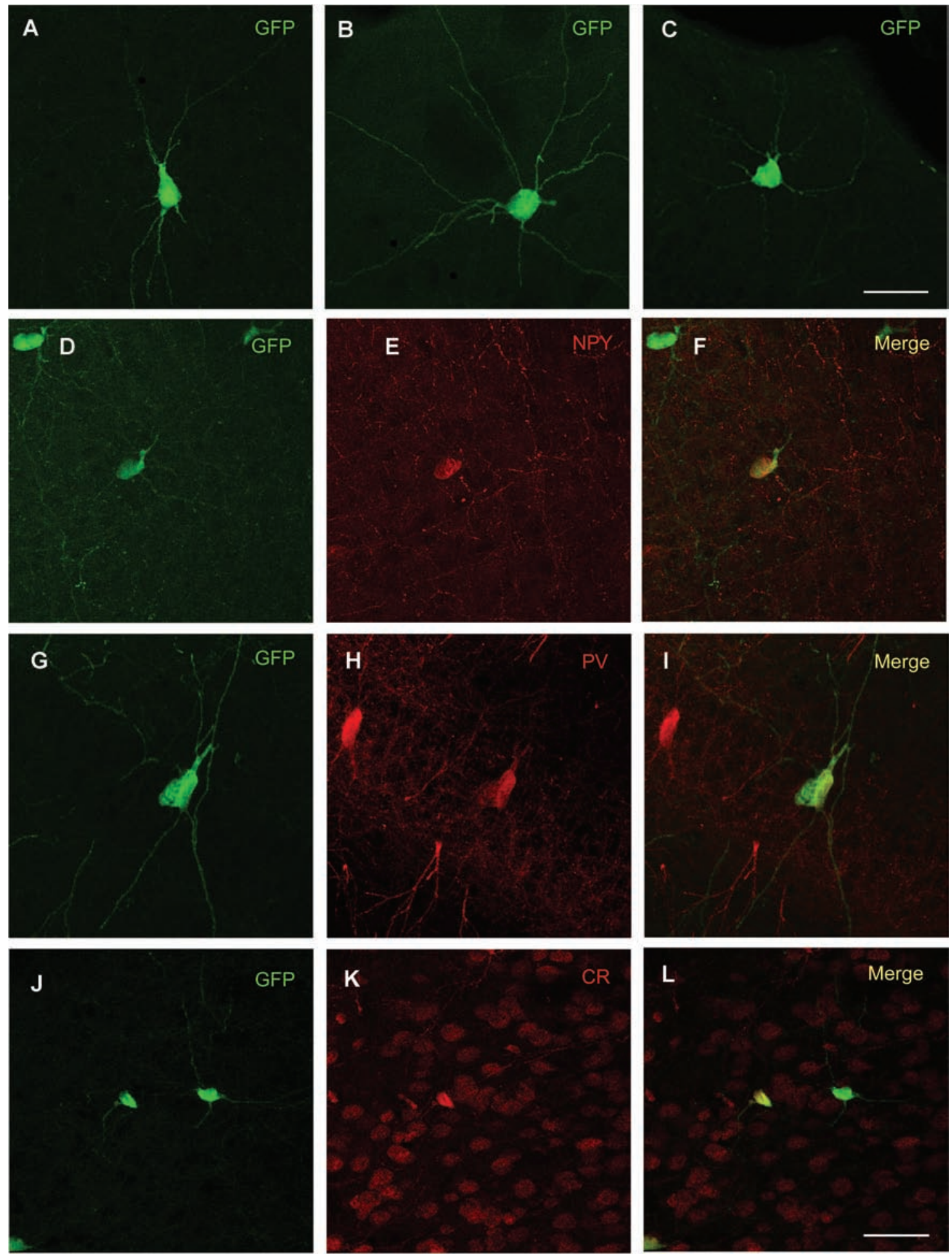

Figure 3. Morphology of MGE-derived $\mathrm{GFP}^{+}$cells 30 days after transplantation. Morphology of $\mathrm{GFP}^{+} \mathrm{MGE}_{\text {graft-derived cells in }}$ different brain areas 30 days posttransplantation in the neocortex (A), striatum (B), and CA1 (C). Confocal images showing colocalization of GFP (green) and interneuronal markers (red); GFP ${ }^{+}$and $\mathrm{NPY}^{+}$in the neocortex (D-F), $\mathrm{GFP}^{+}$and $\mathrm{PV}^{+}$in $\mathrm{CA} 1$ (G-I), and GFP ${ }^{+}$ and $\mathrm{CR}^{+}$in the striatum $(\mathrm{J}-\mathrm{L})$. Merged images $(\mathrm{F}, \mathrm{I}, \mathrm{L})$ indicate double-stained cells, respectively, in the cortex, CA1, and striatum. Scale bars: $30 \mu \mathrm{m}$. 

Table 1. Percentage (\%) of Medial Ganglionic Eminence (MGE)-Derived Cells

\begin{tabular}{lccccc}
\hline & Hippocampus & Neocortex & Striatum & Amygdala & Thalamus \\
\hline $\mathrm{GFP}^{+}$ & 38.7 & 35.4 & 17 & 6.7 & 3 \\
Double-stained cells & 33.3 & 30.8 & 28.3 & 6.3 & 1.26 \\
$\mathrm{GFP}^{+} / \mathrm{NPY}^{+}$ & 37.5 & 34.4 & 25 & 3.1 & 0 \\
$\mathrm{GFP}^{+} / \mathrm{CR}^{+}$ & 40 & 40 & 17.8 & 2.2 & 0 \\
$\mathrm{GFP}^{+} / \mathrm{PV}^{+}$ & 28 & 24.4 & 35.4 & 9.7 & 2.4 \\
\hline
\end{tabular}

Percentage of green fluorescent protein (GFP)-positive and double-stained MGE transplanted cells counted in different areas. All percentages are expressed as the total number of $\mathrm{GFP}^{+}$cells or double-stained interneuronal marker-positive cells counted within the five areas. NPY, neuropeptide Y; CR, calretinin; PV, parvalbumin.

transplantation. This absence of changes in behavior suggests that the cells were in an undifferentiated stage, having a weak functional interaction with the surrounding tissue at this age, as reported previously by electrophysiology experiments (1).

Interestingly, the anxiolytic-like behavior effect began to appear at 30 days, presumably when the transplanted cells integrated into surrounding tissue, and assumed an inhibitory phenotype. In concordance with these findings, previous studies showed a significant increase in inhibitory synapses in cortical slices from transplanted animals that started 30 days after MGE cell transplantation, for example, a time when the interneurons with mature morphology were observed $(1,13)$. De la Cruz and colleagues (13) suggest that MGE cells could secrete GABA and act early on the control of the inhibitory levels in the cortex. These activities could be the reason for the decrease in the anxiety behavior patterns in the MGE groups at 30 and 60 days after transplantation, which is indicated by an increase in the percentage of entries into and the time spent in the open arms.

Our results from the 30- and 60-day groups (MGE30 and MGE60) are consistent with a decrease in anxiety-like behavior described previously by Cunningham and colleagues (11). These authors showed that adult rats tested 6 weeks after the engraftment of cells derived from the lateral ganglionic eminence (LGE) spent more time in the open arms of the EPM compared to CTRL animals. Cells from the LGE have less migratory potential than those from the MGE, and they remained close to the injection site when engrafted directly into the amygdaloid nuclei. As mentioned previously, MGE-transplanted $\mathrm{GFP}^{+}$cells were found in the neocortex, striatum, septal area, hippocampus (CA1 and dentate gyrus), corpus callosum, amygdala, periventricular area, and thalamus. A study of the migration of cortical interneurons in the developing brains of mice suggested that interneurons derived from the MGE showed a wide range of patterns in terms of the speed and direction of migration until they were dispersed throughout the cortex (35).

Over the last two decades, circuits related to the behavior of fear and anxiety have been described, connecting the amygdala with other regions of the brain $(33,36)$. Here, low percentages of MGE cells were found in the amygdala. Nevertheless, the amygdala is not the only structure involved in anxiety and fear conditioning. Structures such as prefrontal cortex, striatum, and hippocampus are also involved $(3,4,6,16,17,21,27)$. Coincidentally, striatum and hippocampus showed high percentages of MGE-derived cells, although they were not the target structures of the injections in the transplantation. The diffuse migration of MGE-derived inhibitory cells throughout the cortex was sufficient to influence the behavior. This high amount of MGE cells within the hippocampus did not produce differences in memory tests. In a recent study, the grafting of neurospheres from the MGE into the hippocampus did not improve learning and memory function in the water maze, although the authors suggested that MGE cells have an anticonvulsant effect (39).

A number of papers have shown the quantification of MGE-derived transplanted cells. Some of these papers used lesion strategies or disease models in which the precise quantification of the cells is crucial data $(5,11-$ $13,39,43)$. However, we must consider the possibility of a lack of correlation of quantitative data of transplanted cells with the observed behavioral response. As proposed by De La Cruz and colleagues, the number of MGE transplanted cells did not correlate with the degree of attenuation of ictal discharges and the protection from seizures found in transplanted animals (13). These results indicate that the mechanism of action of MGE transplants to attenuate ictal power is independent of the transplant cell number.

The presence of $\mathrm{GFP}^{+}$cells expressing GABAergic markers in different areas indicates that MGE cells could migrate throughout the brain parenchyma and differentiate into inhibitory interneurons in vivo. These data confirm the findings of other studies, which explore double labeling of interneurons expressing calretinin, parvalbumin, and NPY in adult animals $(1,9,43)$. The morphological features described for the MGE15 cells indicated the immature nature of the transplanted cells. Moreover, large numbers of $\mathrm{GFP}^{+}$cells could be detected in the corpus callosum in that group, suggesting a migratory pathway of these 
cells during this stage of development. MGE-derived cells expressing interneuronal markers in P15 were rare, presented in clusters (NPY and PV) or scattered (CR) mainly in the cortex. Interestingly, the absence of anxiolytic effects at 15 days after transplantation could be attributed to the undifferentiated stage of the MGE cells at this age. At later time points, 1 and 2 months after transplantation, the majority of the $\mathrm{GFP}^{+}$cells could also be observed in other brain regions, and they expressed interneuronal markers. $\mathrm{GFP}^{+}$cells presented morphological characteristics of inhibitory cells at 30 and 60 days after transplantation (14). In many instances, these cells appeared to project to and possibly connect with each other, as observed in the granular layer and striatum.

As proposed by others, transplanted MGE-derived precursor cells not only differentiated into inhibitory interneurons but also integrated into the preexisting neuronal network. In adult animals, the use of interneuronal precursor cells from the MGE could repair deficits of the inhibitory synaptic function in GABAergic neurotransmission $(1,5,38)$. What could be the in vivo consequences to the animal behavior from this increased inhibition? Our data suggest that precursor cells derived from the MGE and transplanted into neonate animals can change the behaviors that are mediated by GABAergic neurons in adulthood. That scenario can be explained by decreased levels of anxiety-like behavior in the host animal. Our study contributes to a better understanding of GABAergic circuits in anxiety and provides a new approach for future studies on the treatment of disorders in which inhibitory synaptic function is altered.

ACKNOWLEDGMENTS: We are grateful to Henrique Biehl from the Centro de Microscopia Eletrônica of the Universidade Federal do Rio Grande do Sul for confocal technical assistance. This work was supported by CAPES (M.E.C. is a PNPD fellow), FAPESP, and CNPq (Brazil). The authors declare no conflicts of interest.

\section{REFERENCES}

1. Álvarez-Dolado, M.; Calcagnotto, M. E.; Karkar, K.; Southwell, D.; Jones-Davis, D.; Estrada, R.; Rubenstein, J.; Alvarez-Buylla, A.; Baraban, S. Cortical inhibition modified by embryonic neural precursors grafted into the postnatal brain. J. Neurosci. 26(28):7380-7389; 2006.

2. Aroniadou-Anderjaska, V.; Qashu, F.; Braga, M. F. Mechanisms regulating GABAergic inhibitory transmission in the basolateral amygdala: Implications for epilepsy and anxiety disorders. Amino Acids 32(3):305-315; 2007.

3. Bannerman, D. M.; Grubb, M.; Deacon, R. M.; Yee, B. K.; Feldon, J.; Rawlins, J. N. Ventral hippocampal lesions affect anxiety but not spatial learning. Behav. Brain Res. 139(1-2):197-213; 2003.

4. Bannerman, D. M.; Rawlins, J. N.; McHugh, S. B.; Deacon, R. M.; Yee, B. K.; Bast, T.; Zhang, W. N.; Pothuizen, H. H.; Feldon, J. Regional dissociations within the hippocampusmemory and anxiety. Neurosci. Biobehav. Rev. 28(3):273$283 ; 2004$.
5. Baraban, S. C.; Southwell, D. G.; Estrada, R. C.; Jones, D. L.; Sebe, J. Y.; Alfaro-Cervello, C.; García-Verdugo, J. M.; Rubenstein, J. L.; Alvarez-Buylla, A. Reduction of seizures by transplantation of cortical GABAergic interneuron precursors into Kv1.1 mutant mice. Proc. Natl. Acad. Sci. USA 106(36):15472-15477; 2009.

6. Barkus, C.; McHugh, S. B.; Sprengel, R.; Seeburg, P. H.; Rawlins, J. N.; Bannerman, D. M. Hippocampal NMDA receptors and anxiety: At the interface between cognition and emotion. Eur. J. Pharmacol. 626(1):49-56; 2010.

7. Ben-Ari, Y. Excitatory actions of GABA during development: The nature of the nurture. Nat. Rev. Neurosci. 3(9): 728-739; 2002.

8. Ben Ari, Y.; Gaiarsa, J. L.; Tyzio, R.; Khazipov, R. GABA: A pioneer transmitter that excites immature neurons and generates primitive oscillations. Physiol. Rev. 87:12151284; 2007.

9. Calcagnotto, M. E.; Ruiz, L. P.; Blanco, M. M.; SantosJunior, J. G.; Valente, M. F.; Patti, C.; Frussa-Filho, R.; Santiago, M. F.; Zipancic, I.; Álvarez-Dolado, M.; Mello, L. E.; Longo, B. M. Effect of neuronal precursor cells derived from medial ganglionic eminence in an acute epileptic seizure model. Epilepsia 51:71-75; 2010.

10. Carobrez, A. P.; Bertoglio, L. J. Ethological and temporal analyses of anxiety-like behavior: The elevated plus-maze model 20 years on. Neurosci. Biobehav. Rev. 29:1193$1205 ; 2005$.

11. Cunningham, M. G.; Connor, C. M.; Carlezon, Jr., W. A.; Meloni, E. Amygdalar GABAergic-rich neural grafts attenuate anxiety-like behavior in rats. Behav. Brain Res. 205(1): 146-153; 2009.

12. Daadi, M. M.; Lee, S. H.; Arac, A.; Grueter, B. A.; Bhatnagar, R.; Maag, A. L.; Schaar, B.; Malenka, R. C.; Palmer, T. D.; Steinberg, G. K. Functional engraftment of the medial ganglionic eminence cells in experimental stroke model. Cell Transplant. 18(7):815-826; 2009.

13. De la Cruz, E.; Zhao, M.; Guo, L.; Ma, H.; Anderson, S. A.; Schwartz, T. H. Interneuron progenitors attenuate the power of acute focal ictal discharges. Neurotherapeutics 8(4): 763-773; 2011.

14. Freund, T. F.; Buzsáki, G. Interneurons of the hippocampus. Hippocampus 6(4):347-470; 1996.

15. Frussa-Filho, R.; Ribeiro, R. A. One-trial tolerance to the effects of chlordiazepoxide in the elevated plus-maze is not due to acquisition of a phobic avoidance of open arms during initial exposure. Life Sci. 71(5):519-525; 2002.

16. Fuss, J.; Ben Abdallah, N. M.; Hensley, F. W.; Weber, K. J.; Hellweg, R.; Gass, P. Deletion of running-induced hippocampal neurogenesis by irradiation prevents development of an anxious phenotype in mice. PLoS One 5(9):e12769; 2010.

17. Grafman, J.; Vance, S. C.; Weingartner, H.; Salazar, A. M.; Amim, D. The effects of lateralized frontal lesions on mood regulation. Brain 109:1127-1148; 1986.

18. Handley, S. L.; Mithani, S. Effects of alpha-adrenoceptor agonists and antagonists in a maze-exploration model of 'fear'-motivated behaviour. Naunyn Schmiedebergs Arch. Pharmacol. 327(1):1-5; 1984.

19. Hikosaka, O. GABAergic output of the basal ganglia. Prog. Brain Res. 160:209-226; 2007.

20. Hogg, S. Review of the validity and variability of the elevated plus-maze as an animal model of anxiety. Pharmacol. Biochem. Behav. 54(1):21-30; 1996.

21. Holmes, A.; Quirk, G. J. Pharmacological facilitation of fear extinction and the search for adjunct treatments for anxiety 
disorders - the case of yohimbine. Trends Pharmacol Sci. 31(1):2-7; 2010.

22. Kluver, H.; Bucy, P.C. Preliminary analysis of functions of the temporal lobes in monkeys. 1939. J. Neuropsychiatry Clin. Neurosci. 9(4):606-620; 1997.

23. Lister, R. G. Ethologically-based animal models of anxiety disorders. Pharmacol. Ther. 46(3):321-340; 1990.

24. Makkar, S. R.; Zhang, S. Q.; Cranney, J. Behavioral and neural analysis of GABA in the acquisition, consolidation, reconsolidation, and extinction of fear memory. Neuropsychopharmacology 35(8):1625-1652; 2010.

25. Marin, O.; Rubenstein, J. L. Along, remarkable journey: Tangential migration in the telencephalon. Nat. Rev. Neurosci. 2:780-790; 2001.

26. Martínez-Cerdeño, V.; Noctor, S. C.; Espinosa, A.; Ariza, J.; Parker, P.; Orasji, S.; Daadi, M. M.; Bankiewicz, K.; Alvarez-Buylla, A.; Kriegstein, A. R. Embryonic MGE precursor cells grafted into adult rat striatum integrate and ameliorate motor symptoms in 6-OHDA-lesioned rats. Cell Stem Cell 6(3):238-250; 2010.

27. McNaughton, N.; Gray, J. A. Anxiolytic action on the behavioural inhibition system implies multiple types of arousal contribute to anxiety. J. Affect. Disord. 61(3):161$176 ; 2000$.

28. Nemeroff, C. B. The role of GABA in the pathophysiology and treatment of anxiety disorders. Psychopharmacol. Bull. 37(4):133-146; 2003.

29. Paxinos, G.; Franklin, K. B. J. The Mouse Brain in Stereotaxic Coordinates, 2nd ed. San Diego, CA: Academic Press; 2001.

30. Pellow, S.; Chopin, P.; File, S. E.; Briley, M. Validation of open:closed arm entries in an elevated plus-maze as a measure of anxiety in the rat. J. Neurosci. Methods 14(3):149$167 ; 1985$.

31. Rodgers, R. J. Animal models of 'anxiety': Where next? Behav. Pharmacol. 8(6-7):477-496; 1997.

32. Sandford, J. J.; Argyropoulos, S. V.; Nutt, D. J. The psychobiology of anxiolytic drugs. Part 1: Basic neurobiology. Pharmacol. Ther. 88(3):197-212; 2008.

33. Schulkin, J. Angst and the amygdala. Dialogues Clin. Neurosci. 8(4):407-416; 2006.
34. Silva, R. H.; Kameda, S. R.; Carvalho, R. C.; TakatsuColeman, A. L.; Niigaki, S. T.; Abílio, V. C.; Tufik, S.; Frussa-Filho, R. Anxiogenic effect of sleep deprivation in the elevated plus-maze test in mice. Psychopharmacology 176:115-122; 2004

35. Tanaka, D. H.; Yanagida, M.; Zhu, Y.; Mikami, S.; Nagasawa, T.; Miyazaki, J.; Yanagawa, Y.; Obata, K.; Murakami, F. Random walk behavior of migrating cortical interneurons in the marginal zone: Time-lapse analysis in flat-mount cortex. J. Neurosci. 29:1300-1311; 2009.

36. Tasan, R. O.; Nguyen, N. K.; Weger, S.; Sartori, S. B.; Singewald, N.; Heilbronn, R.; Herzog, H.; Sperk, G. The central and basolateral amygdala are critical sites of neuropeptide Y/Y2 receptor-mediated regulation of anxiety and depression. J. Neurosci. 30(18):6282-6290; 2010.

37. Taubenfeld, S. M.; Wiig, K. A.; Bear, M. F.; Alberini, C. M. A molecular correlate of memory and amnesia in the hippocampus. Nat. Neurosci. 2(4):309-310; 1999.

38. Thompson, K. Transplantation of GABA-producing cells for seizure control in models of temporal lobe epilepsy. Neurotherapeutics 6(2):284-294; 2009.

39. Waldau, B.; Hattiangady, B.; Kuruba, R.; Shetty, A. K. Medial ganglionic eminence-derived neural stem cell grafts ease spontaneous seizures and restore GDNF expression in a rat model of chronic temporal lobe epilepsy. Stem Cells 28(7):1153-1164; 2010.

40. Wichterle, H.; Turnbull, D. H.; Nery, S.; Fishell, G.; AlvarezBuylla, A. In utero fate mapping reveals distinct migratory pathways and fates of neurons born in the mammalian basal forebrain. Development 128:3759-3771; 2001.

41. Wilson, S. W.; Rubenstein, J. L. Induction and dorsoventral patterning of the telencephalon. Neuron 28(3):641$651 ; 2000$.

42. Wonders, C. P.; Anderson, S. A. The origin and specification of cortical interneurons. Nat. Rev. Neurosci. 7(9):687696; 2006

43. Zipancic, I.; Calcagnotto, M. E.; Piquer-Gil, M.; Mello, L. E.; Álvarez-Dolado, M. Transplant of GABAergic precursors restores hippocampal inhibitory function in a mouse model of seizure susceptibility. Cell Transplant. 19(5):549-564; 2010. 